\title{
From Caging to Grasping
}

\author{
Alberto Rodriguez \\ The Robotics Institute \\ Carnegie Mellon University \\ Pittsburgh, PA 15206 \\ Email: albertor@cmu.edu
}

\author{
Matthew T. Mason \\ The Robotics Institute \\ Carnegie Mellon University \\ Pittsburgh, PA 15206 \\ Email: matt.mason@cs.cmu.edu
}

\author{
Steve Ferry \\ Department of Mathematics \\ Rutgers University \\ Piscataway, NJ 08854-8019 \\ Email: sferry@math.rutgers.edu
}

\begin{abstract}
This paper digs into the relationship between grasps and cages of a rigid body, in particular into the use of cages as waypoints to grasp an object. We introduce the concept of pregrasping cages, caging configurations from which the object can be reached while maintaining the cage on it. In the well understood case of two-fingered manipulators, the squeezing/stretching caging characterization implies that all cages are indeed pregrasping cages and, as a consequence, are useful waypoints to grasp an object. We show in this paper that the same does not hold for more than two fingers. There are caging configurations from which a grasp of the object cannot be reached without breaking the cage on it. We explore the natural generalization of the squeezing/stretching characterization to the case of $n$ fingers and exploit it to give sufficient conditions for a cage to be a pregrasping cage.
\end{abstract}

\section{INTRODUCTION}

To cage an object is to bound its mobility. From the object's point of view, the manipulator constitutes a set of constraints in the object's configuration space that prevents it from escaping arbitrarily far.

In opposition to the prehensile approach to manipulation, caging provides a way to control an object without immobilizing it. When manipulating an object, a cage on it is enough to guarantee that the object will "follow" while the manipulator moves as a rigid body. Non-prehensile manipulation is the most widely referenced application of caging [1]-[3].

Caging is also interesting for a second reason: When an immobilizing grasp is required, a cage might constitute a useful waypoint to the grasp. In some cases, the grasping problem becomes trivial once the caging problem is solved. It is on this less referenced application of caging - the relationship between grasping and caging configurations- that we set the focus of this paper.

Best studied is the case of two-fingered manipulators. The introduction of squeezing and stretching conditions by Vahedi and van der Stappen [4] has allowed for a deep understanding of their configuration space: All caging configurations classify either as configurations that remain caged when the fingers are given the freedom of moving closer together (squeezing) and/or configurations that remain caged when the fingers are given the freedom of moving apart (stretching), Fig. 1.

This characterization gives rise to an interesting relationship between cages and grasps. Given a cage of an object, there is always an infallible blind strategy to grasp it: either close or open the fingers, depending on whether the cage is a squeezing

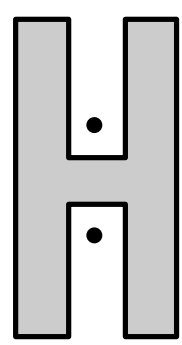

(a)

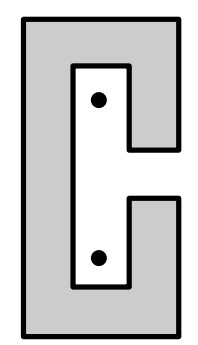

(b)

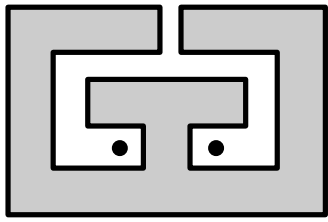

(c)
Fig. 1. Examples of (a) squeezing caging, (b) stretching caging (c), both.

or a stretching cage. No need for accurate finger positioning or feedback for error control. The squeezing/stretching characterization guarantees that the object will not be able to escape while we close/open the fingers. In this paper we refer to any cage with such grasping strategy as pregrasping cage.

Is it possible to extend the same characterization to $n$ fingered manipulators? Can we always infallibly grasp an object beginning from a cage? The answer to the first question is yes. This paper generalizes the squeezing/stretching characterization from two-fingered cages to an arbitrary number of fingers. Unfortunately, the answer to the second question is no. In general, not all cages are an adequate waypoint to grasp an object, that is, not all cages are pregrasping cages.

Section II describes previous work on caging and its relation to grasping. In Sect. III we give precise definitions of concepts related to configuration space, caging, and grasping.

We discuss the relationship between caging and grasping in Sect. IV and continue with a brief exposition of the two-fingered case in Sect. V. Section VI gives a thorough exploration of the generalization to the case of $n$ fingers.

A consequence of the proposed generalization is that, by maintaining the value of certain scalar functions defined on the finger formation, we can give partial freedom to the point fingers while guaranteeing the cage on the object. In addition, if the manipulator forms an appropriate pregrasping cage, we can easily grasp an object by actively controlling the value of that function. Sect. VII shows three examples of such functions. We finish in Sect. VIII with a review and discussion of the implications of the presented generalization. 


\section{RELATED WORK}

Kuperberg [5] was the first to propose a formal definition for the caging problem:

"Let $P$ be a polygon in the plane, and let $C$ be a set of $n$ points in the complement of the interior of $P$. The points capture $P$ if $P$ cannot be moved arbitrarily far from its original position without at least one point of $C$ penetrating the interior of $P$. Design an algorithm for finding a set of capturing points for $P$."

Since then, the caging condition has been studied from different perspectives and applied to a number of manipulation problems such as grasping [6], part feeding [2], and nonprehensile manipulation [1], [7].

Relevant to our work are the concepts of squeezing and stretching caging proposed in recent algorithmic approaches to the two-finger caging problem [4], [8]. Squeezing and stretching caging are stricter conditions than caging that expect the object's mobility to be bounded even when the manipulator is allowed some partial freedom.

Rimon and Blake [9] were the first to implicitly study squeezing cages. They were interested in determining the caging set of a grasp: the maximal connected set of caging configurations containing a given immobilization of the object. They showed that the hand's configuration at which the cage breaks always corresponds to a frictionless equilibrium grasp, and used that characterization to develop caging algorithms for two-fingered gripping systems. Although they only explicitly considered external or squeezing grasps, they already are interested in the connection between immobilizations of the object and caging configurations.

Pipattanasomporn and Sudsang's work on caging concave polygons with two fingers [8] had a similar goal as Rimon and Blake's but with an algorithmic rather than topological approach. They studied the close problem of finding maximal two-fingered cages on concavities of planar polygons. They introduced the concepts of equivalent squeezed and stretched finger trajectories that allowed them to transform the caging problem into a graph search problem in a finite discrete space.

The concepts of squeezing and stretching caging were formally developed by Vahedi and van der Stappen [4] as an algorithmic technique for the problem of finding all twofingered caging placements of planar polygons. They showed that, under those circumstances, squeezing and stretching cages accounted for all possible cages and used it to develop the first complete algorithm to report them.

Rodriguez and Mason [10] later generalized the squeezing/stretching classification of two-fingered cages to all compact, connected and contractible objects in arbitrary dimension, suggesting that such characterization is a fundamental attribute of the configuration space of two-fingered manipulators. The discussion presented in this paper builds on the basis of a further generalization of Rodriguez and Mason's result to the case of $n$-fingered manipulators.
One of the main ideas expressed in this paper is that certain caging configurations can be used as waypoints to reliably grasp an object. The closest work in the literature is that of error-tolerant grasping techniques for two-fingered manipulators developed by Davidson and Blake [11] and Gopalakrishnan and Goldberg [12]. Both use, in some way or another, the notion of caging to robustify the grasping process.

We also find in the literature works relating to the study of caging with an arbitrary number of fingers. An early example is that by Pereira, Campos and Kumar [1] on conservative decentralized control strategies to maintain caging in multirobot formations for manipulation of planar objects.

Most relevant to our work is that of Pipattanasomporn, Vogmasa and Sudsang [13] on caging polytopes via finger dispersion control. They proposed to cage polytopes by maintaining a scalar function defined on the finger formation over a critical value. Our work can be seen as a formalization and extension of the same idea for general objects. We present it here as the natural generalization to $n$ fingers of the squeezing/stretching characterization of two finger cages.

\section{PRELIMINARY CONCEPTS}

Here we present concepts and definitions used in the paper in relation to the configuration space, caging, and grasping.

\section{A. Configuration Space}

Let the manipulator be a set of point fingers $p_{1} \ldots p_{n}$ in the workspace $\mathcal{W} \simeq \mathbb{R}^{d}$, and let $\mathcal{M}=\mathcal{W}_{p_{1}} \times \ldots \times \mathcal{W}_{p_{n}} \simeq\left(\mathbb{R}^{d}\right)^{n}$ be the configuration space of the manipulator.

Let $\mathcal{O} \subset \mathcal{W}$ be the object to manipulate. We will only consider objects homeomorphic to an Euclidean closed ball in $\mathcal{W}$, and will refer to as topological ball. We note by $\mathcal{O}^{M}=$ $\left\{\left(p_{1} \ldots p_{n}\right) \in \mathcal{M} \mid \exists p_{i} \in \mathcal{O}\right\}$ the configuration space obstacle induced by $\mathcal{O}$. Following the convention in [4], [10], [14] the free workspace of each point finger $p_{i}$ is defined as the complement of the interior of $\mathcal{O}, \mathcal{W}_{p_{i}}^{\text {free }}=\mathcal{W}_{p_{i}} \backslash$ int $[\mathcal{O}]$, and equivalently the free space of the manipulator as $\mathcal{M}^{\text {free }}=$ $\mathcal{M} \backslash$ int $\left[\mathcal{O}^{M}\right]$.

An admissible path of the manipulator is a parametrized curve $\alpha:[0,1] \longrightarrow \mathcal{M}^{\text {free }}$. A contractible path is an admissible closed path homotopic to a point $c$ in $\mathcal{M}^{\text {free }}$, which holds subject to the existence of a homotopy of paths:

$$
H(t, s):[0,1] \times[0,1] \longrightarrow \mathcal{M}^{\text {free }}
$$

with $H(t, 0)=\alpha(t)$ and $H(t, 1)=c$.

Finally we introduce the natural projection operator $\Pi_{i}$ : $\mathcal{M} \longrightarrow \mathcal{W}_{p_{i}}$ that projects a configuration of the manipulator $c=\left(p_{1} \ldots p_{n}\right)$ into the $i$-th coordinate $p_{i}$.

\section{B. Caging}

A cage is a configuration $c$ of the manipulator that bounds the mobility of the object. In Kuperberg's original formulation of the caging problem [5], point fingers $p_{1} \ldots p_{n}$ are interpreted as obstacles in the configuration space of the object $\mathcal{O}$ meant to bound the set of its free paths. In this paper, as it has been the rule in the literature on caging [4], [10], [13], 
[14], we use the equivalent and more convenient definition in terms of the mobility of the manipulator.

Given a configuration $c \in \mathcal{M}$ of the manipulator, let the rigid body subspace $\mathcal{M}_{c}$ be the set of all configurations of the manipulator obtained by rigid transformations from $c$. A cage or caging configuration is then defined as:

Definition 1 (Caging Configuration). Configuration $c$ of the manipulator that lies in a compact connected component of $\mathcal{M}^{\text {free }} \cap \mathcal{M}_{c}$.

Consequently, if the manipulator does not cage the object, there must be an escaping path in $\mathcal{M}^{\text {free }} \cap \mathcal{M}_{c}$ that brings the manipulator arbitrarily far from $\mathcal{O}$.

\section{Grasping}

We show in this paper that if we start from a suitable caging configuration, we can be certain of grasping an object while following a simple control strategy. Before getting into any details of that process, we specify here what we mean by grasp.

First, we review the concept of equilibrium grasp: Given a set of point fingers $p_{1} \ldots p_{n}$ in contact with the object, and their correspondent contact normals $\boldsymbol{\eta}_{1} \ldots \boldsymbol{\eta}_{n}$, we say that the manipulator holds a frictionless equilibrium grasp on the object if there is a positive linear combination $\lambda_{1} \boldsymbol{\eta}_{1}+$ $\ldots \lambda_{n} \boldsymbol{\eta}_{n}=\mathbf{0}$ with $\lambda_{i} \geq 0$ and not all $\lambda_{i}=0$.

We will refer to the type of grasp that we expect to get as grasping cage, and define it as:

Definition 2 (Grasping Cage). Caging configuration $c$ of the manipulator for which a subset of the fingers holds an equilibrium grasp on the object.

Notice that the definition is not as constraining as an immobilization of the object, but is stricter than a regular equilibrium grasp in the sense that even if the equilibrium becomes unstable, the manipulator still cages the object and will not be able to escape its "grip".

\section{FROM CAGING TO GRASPING}

In this section we put forward the idea that not all cages are equally suited for the purpose of grasping an object. The first step in that direction is to clearly state what it means for a cage to facilitate the grasping process. With that in mind, we introduce the concept of pregrasping cage:

Definition 3 (Pregrasping Cage). Caging configuration $c$ of the manipulator that yields the existence of a caging path to a grasping cage of the object.

Pregrasping cages are configurations of the manipulator from where the object can be reached without breaking the cage on it. There is a grasping strategy that yields absolute certainty about the object never escaping the manipulator.

Figure 2 shows a simple example of a pregrasping cage with two fingers. Beginning from a caging configuration, the manipulator closes the fingers to grasp the object never loosing the cage on it and, by definition, reaching a grasping cage. We will review in Sect. V that this is always the case when the manipulator is composed of two fingers.

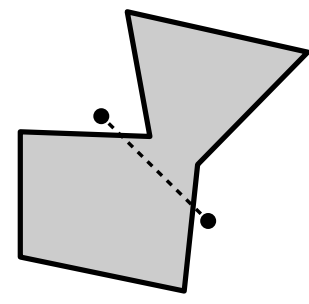

(a)

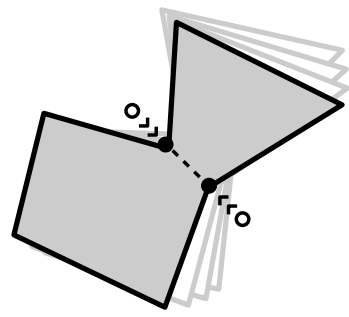

(b)
Fig. 2. (a) Example of a pregrasping cage with two fingers. (b) While moving the fingers closer together, the manipulator never breaks the cage on the object.

The same idea can be extended to grasping with multifingered manipulators. Figure 3 shows an example where a set of three fingers close simultaneously from a caging configuration to reach a grasp. The manipulator cages the object throughout the entire grasping process.

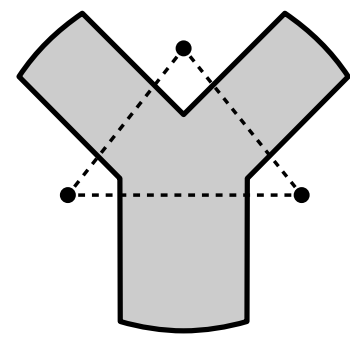

(a)

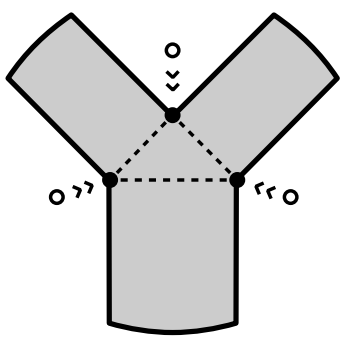

(b)
Fig. 3. (a) Example of a multifingered pregrasping cage, (b) During the process of bringing the fingers together, the object remains caged.

A key observation is that in the general case, unlike in the case of two fingers, the set of pregrasping cages is a proper subset of the set of all cages. Figure 4 shows an example of a cage that cannot be extended to a grasp without breaking loose of the cage. In the same figure we illustrate that fact by showing that a slightly larger triad of point fingers can actually escape.

We are interested in identifying pregrasping cages and their associated grasping strategies. In Section VI we show how we can capitalize on the generalization to multifingered manipulators of the squeezing/stretching motions and identify sets of pregrasping cages.

\section{Two Finger Caging: SqueEzing And Stretching}

In this section we show how all cages of a two-fingered manipulator are pregrasping cages. The discussion is based on the squeezing/stretching characterization by Vahedi and van der Stappen [4] of all cages of a planar polygon and on Rodriguez and Mason's extension to any compact connected and contractible object of arbitrary dimension [10]. The topological approach used by Rodriguez and Mason, briefed in this 


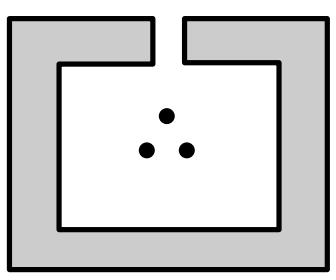

(a)
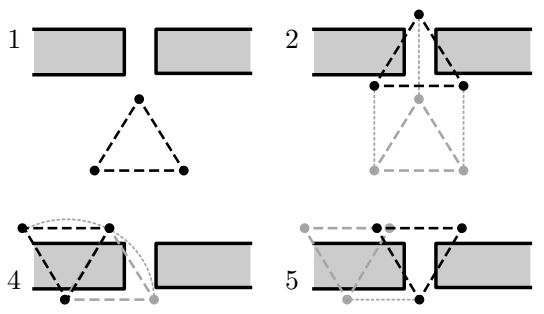

(c)

Fig. 4. (a) Example of a cage with three fingers that is not a pregrasping cage (b) As soon as the fingers stretch to reach a grasp they get to configurations like this one where the object is no longer trapped. (c) Step by step escaping path that shows that the object is actually not trapped in (b).

section, is key for the generalization to the case of $n$ fingers in Sect. VI. This section should serve as a guide to better understand the posterior generalization.

Intuitively, an object is squeezing caged if it is trapped even if the point fingers are given the freedom of moving closer together. Contrarily, it is stretching caged if the object remains trapped even when the fingers are given the freedom to move apart, Fig. 1.

\section{A. The Squeezing and Stretching Characterization}

Let $D: \mathcal{M} \rightarrow \mathbb{R}$ be the distance between the two fingers. Then the rigid body subspace introduced in Sect. III-B can be redefined as $\mathcal{M}_{c}=\{q \in \mathcal{M} \mid D(q)=D(c)\}$. Note that $\mathcal{M}_{c}$ is the preimage of a smooth function. This interpretation is crucial for the generalization to the $n$ finger case.

We now formally define the concepts of squeezing and stretching caging. Given a configuration of the manipulator $c$, let $\mathcal{M}_{c}^{+}$and $M_{c}^{-}$be the subsets:

$$
\begin{aligned}
& \mathcal{M}_{c}^{+}=\{q \in \mathcal{M} \mid D(q) \geq D(c)\} \\
& \mathcal{M}_{c}^{-}=\{q \in \mathcal{M} \mid D(q) \leq D(c)\}
\end{aligned}
$$

Analogously to the definition of a caging configuration in Sect. III-B, we define a squeezing (stretching) cage as:

Definition 4 (Squeezing (Stretching) Caging). Configuration $c$ of the manipulator that lies in a compact connected component of $\mathcal{M}^{\text {free }} \cap \mathcal{M}_{c}^{-}\left(\mathcal{M}^{\text {free }} \cap \mathcal{M}_{c}^{+}\right)$.

The next theorem, from [10], gives the already mentioned squeezing/stretching characterization of two finger cages:
Theorem 1 (Squeezing/Stretching Caging). Given a two finger caging configuration of a topological ball in $\mathbb{R}^{d}$, it is squeezing caging, stretching caging or both.

The proof follows the contrapositive: Suppose that a configuration $c$ of the manipulator is neither squeezing nor stretching caging. By definition, there are squeezing and stretching escaping paths $\alpha^{-} \subset \mathcal{M}_{c}^{-}$and $\alpha^{+} \subset \mathcal{M}_{c}^{+}$that go from $c$ to noncaging configurations $c^{+}$and $c^{-}$arbitrarily far from $c$. The key idea, illustrated in Fig. 5, is to join those escaping paths in the noncaging region of the space to construct a contractible curve. Its contraction yields a path $\alpha_{0}$ in $\mathcal{M}_{c}$ from $c$ to a noncaging configuration $c_{0}$, establishing the noncageness of $c$.
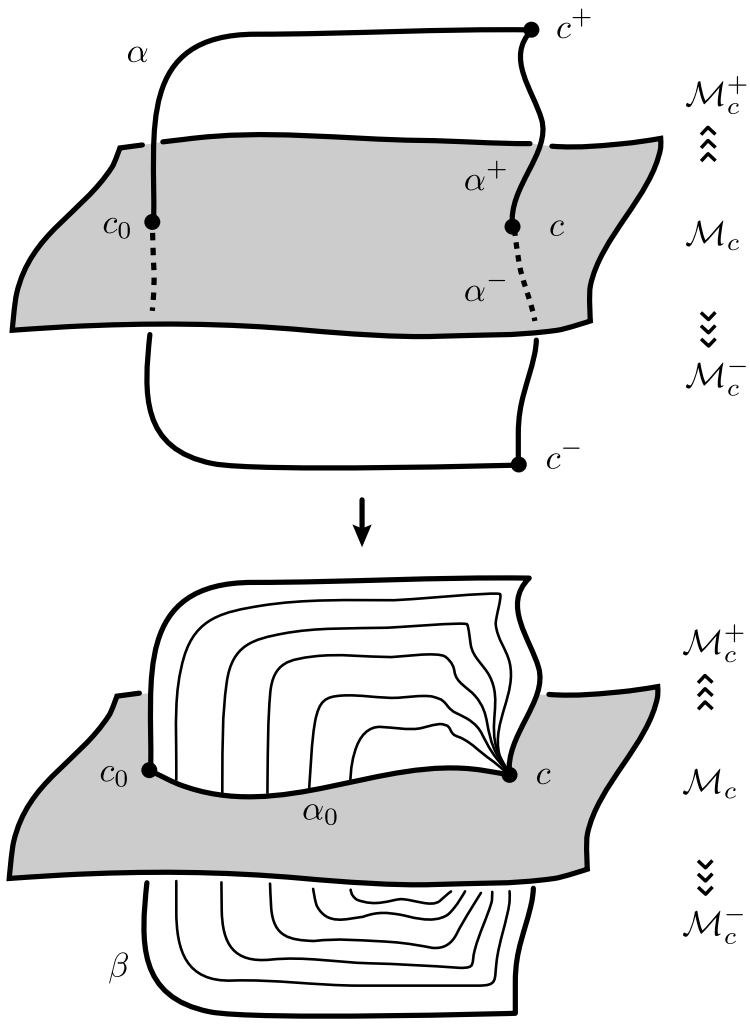

Fig. 5. Diagram of the proof of Theorem 1. The contraction of the curve $\beta=\alpha^{+} \oplus \alpha \oplus \alpha^{-}$gives the path $\alpha_{0}$ in $\mathcal{M}_{c}$ that connects $c$ with a noncaging configuration $c_{0}$.

Although the details are laborious, the idea is simple. In Sect. VI we use the same strategy to study the $n$ finger case.

\section{B. Implications}

By virtue of Theorem 1 all two finger cages can be classified either as squeezing or stretching. That is, if we close the fingers (in the case of a squeezing cage) or open them (in the case of a stretching cage) we will never lose the object.

Both motions, opening and closing, are bounded. Opening because, by definition, the manipulator moves in a compact connected component of $\mathcal{M}^{\text {free }} \cap \mathcal{M}_{c}^{-}$, hence the fingers cannot open infinitely. Closing because when the distance between the 
fingers reaches zero, the manipulator becomes a single point in the workspace which cannot cage the object, given that it is contractible.

When the fingers cannot close (squeezing cage) or open (stretching cage) any more, they both must be in contact with the object with opposing normals and, by definition, in a grasping cage. Hence, all two finger cages are pregrasping cages.

\section{N FINGER CAGING: $F$-SQUEEZING AND $F$-STRETCHING}

The formalization of the concepts of caging, squeezing caging and stretching caging for the case of two fingers depends on the function distance between the fingers $D(c)$, a function defined on the "shape" of the manipulator. Analogously, in the $n$ finger case, we consider functions $F: \mathcal{M} \rightarrow \mathbb{R}$ that are invariant with respect to rigid transformations of the manipulator. We will say that $F$ is defined on the finger formation. As in the case of two fingers, we will consider the level sets $\mathcal{M}_{k}=F^{-1}(k)$.

Given a configuration $c$, we define an $F$-caging configuration or simply $F$-cage as:

Definition 5 ( $F$-Caging Configuration). Configuration $c$ of the manipulator that lies in a compact connected component of $\mathcal{M}^{\text {free }} \cap \mathcal{M}_{k}$, with $k=F(c)$.

The manipulator is $F$-caged if and only if it cannot escape when it has the freedom of moving while $F$ maintains its value. As a result, $F$-cageness prevents from the existence of escaping paths in $\mathcal{M}_{k}$.

Notice that the definition of $F$-cage is equivalent to the definition of a two finger cage when the function $F$ is the distance between them. Contrary to the two finger case, when $n \neq 2$, an $F$-cage has a stricter definition than a cage. We will see in Sect. VI-A that, assuming $F$ satisfies a set of properties, all $F$-cages are constrained to be pregrasping cages.

\section{A. The F-Squeezing and F-Stretching Characterization}

We introduce now the concepts of $F$-squeezing cage and $F$ stretching cage analogous to those of squeezing and stretching cages. Given a configuration of the manipulator $c$, let $\mathcal{M}_{k}^{+}$and $\mathcal{M}_{k}^{-}$be the halfspaces:

$$
\begin{aligned}
& \mathcal{M}_{k}^{+}=\{c \in \mathcal{M} \mid F(c) \geq k\} \\
& \mathcal{M}_{k}^{-}=\{c \in \mathcal{M} \mid F(c) \leq k\}
\end{aligned}
$$

We then define $F$-squeezing and $F$-stretching caging as:

Definition 6 ( $F$-Squeezing ( $F$-Stretching) Caging). Configuration $c$ of the manipulator that lies in a compact connected component of $\mathcal{M}^{\text {free }} \cap \mathcal{M}_{k}^{-}\left(\mathcal{M}^{\text {free }} \cap \mathcal{M}_{k}^{+}\right)$, with $k=F(c)$.

The manipulator is in an $F$-squeezing ( $F$-stretching) caging configuration if and only if it cannot escape even when it is given the freedom of moving while maintaining the value of $F$ below (above) its initial value $F(c)$. In Sect. VII we show three examples of functions $F$ with corresponding $F$-squeezing and $F$-stretching cages.
Notice again that the definitions of $F$-squeezing cage and $F$ stretching cage are equivalent to the regular definitions of two finger squeezing and stretching cages when $F$ is the distance between them.

The same way that squeezing and stretching cages characterize the set of all cages of a two-fingered manipulator, the next theorem characterizes the set of all $F$-cages in terms of $F$-squeezing and $F$-stretching cages.

Theorem 2 ( $F$-Squeezing/ $F$-Stretching Caging). Let $F$ be a semialgebraic function defined on the point finger formation. Then, given an F-caging configuration of a topological ball in $\mathbb{R}^{d}$ it is either F-squeezing caging, F-stretching caging or both.

Refer to Appendix A for the proof. Notice that Theorem 1 follows directly from Theorem 2 since the distance between two fingers is a semialgebraic function. Semialgebraicity of $F$ is a sufficient requirement we use to avoid degeneracies in the level sets $\mathcal{M}_{k}$ and simplify the proof of the theorem. However, we do not mean to imply its necessity.

\section{B. Implications}

The next corollary gives the relation between $F$-cages and pregrasping cages:

Corollary 1. Let $F$ be a semialgebraic function defined on the finger formation such that $F^{-1}([-\infty, m])$ and $F^{-1}([M, \infty])$ are nonempty subsets of the non-F-caging region of the configuration space for some $m<M$. Then if the object $\mathcal{O}$ has a piecewise smooth boundary, all $F$-cages are pregrasping cages.

Proof: Following Theorem 2, given that $F$ is semialgebraic, all $F$-cages are either $F$-squeezing or $F$-stretching cages. Consequently, when controlling the finger formation to decrease ( $F$-squeezing) or increase ( $F$-stretching) the value of $F$ the object should remain $F$-caged.

If we monotonically decrease (increase) the value of $F$, due to the fact that $F^{-1}(m)$ and $F^{-1}(M)$ are non- $F$-caging configurations, both motions must be bounded. $F$ cannot grow larger than $M$ or smaller than $m$ because the manipulator would get to a non- $F$-caging configuration, which is prevented by hypothesis.

When $F$ reaches a local minimum $c$ (maximum) and cannot be decreased (increased) any more, the free space is contained in $\mathcal{M}_{c}^{+}\left(\mathcal{M}_{c}^{-}\right)$. A change in the topology of the level sets $\left\{F^{-1}(k)\right\}$ occurs locally at $c$. By an argument similar to the proof of Proposition 1 in [9] we can show that vector $\mathbf{0}$ can then be written as a positive linear combination of the contact normals and consequently $c$ is an equilibrium grasp.

Notice that, besides some regularity conditions, the only requirement $F$ needs to satisfy is that both when its value gets arbitrarily small or large it must not $F$-cage the object.

The process by which the manipulator reaches the grasping cage is less determined than in the case of two fingers, where the shape space has dimension one. Here the manipulator has 
several extra freedoms. The key idea is that any motion of the fingers that implies a corresponding decrease/increase of the value of $F$ will end up with the manipulator grasping the object. In the final configuration, not all fingers need to be in contact with the object and be part of the grasping cage.

\section{EXAMPLES OF FUNCTIONS ON THE FINGER FORMATION}

In this section we show three examples of functions on the finger formation that satisfy the conditions in Corollary 1, and how their values can be controlled to grasp the object.

By definition $F$ must be invariant with respect to rigid transformations of the manipulator. In the following examples we satisfy that condition by making $F$ a function of the distances between the point fingers.

$$
\begin{aligned}
F_{e} & =\frac{1}{2} \sum_{\substack{i, j \in 1 \ldots n \\
i \neq j}} d^{2}\left(p_{i}, p_{j}\right) \\
F_{\max } & =\max _{i, j \in 1 \ldots n} d\left(p_{i}, p_{j}\right) \\
F_{\text {min }} & =\min _{i, j \in 1 \ldots n} d\left(p_{i}, p_{j}\right)
\end{aligned}
$$

The set of induced $F$-cages and therefore the subset of pregrasping cages, depends on the selection of $F$.

All three $F_{e}, F_{\max }$ and $F_{\min }$ are semialgebraic as they are expressed in terms of sums, multiplications and inequalities. Regarding the behavior when they grow small or large:

$F_{e}$ If $F_{e}=0$, all point fingers are coincident, thus, the manipulator neither cages the object nor $F_{e}$-cages it, since the object is contractible and does not have holes. If $F_{e}$ is larger than $\frac{n \cdot(n-1)}{2}$ times the squared diameter of the object, it cannot $F_{e}$-cage it either, since the manipulator is allowed to move freely while maintaining the value of $F_{e}$ until the distance between every pair of points is at least the diameter of the object.

$F_{\max }$ If $F_{\max }=0$ the manipulator becomes a point and it cannot $F_{\max }$-cage the object. If $F_{\max }$ is larger than the diameter of the object, all interdistances between the finger points can grow freely up to the diameter while $F_{\max }$ maintains its value. Hence, the object is not $F_{\max }$-caged either.

$F_{\min }$ If $F_{\min }=0$, all finger points except two that coincide have complete freedom for $F_{\min }$ to maintain its value. The object cannot be $F_{\min }$-caged. If $F_{\min }$ is larger than the diameter of the object, all interdistances between finger points are, by definition, larger than the diameter of the object, thus, it cannot be $F_{m i n}$-caged either.

Figure 6 shows examples of $F$-cages with $F_{e}, F_{\max }$, and $F_{\min }$. Examples $6 \mathrm{a}$ and $6 \mathrm{~b}$ are objects that require more than two fingers to be caged. Example $6 \mathrm{c}$ corresponds to the counterexample in Fig. 4.

We call $F_{e}$ the energy of the finger formation because of the resemblance to the form of the elastic energy that stored by virtual springs connecting the point fingers. We can maintain a cage over an object by controlling that energy level.

$F_{\max }$ and $F_{\min }$ are well suited to be controlled in a decentralized manner. They resemble the conservative decentralized
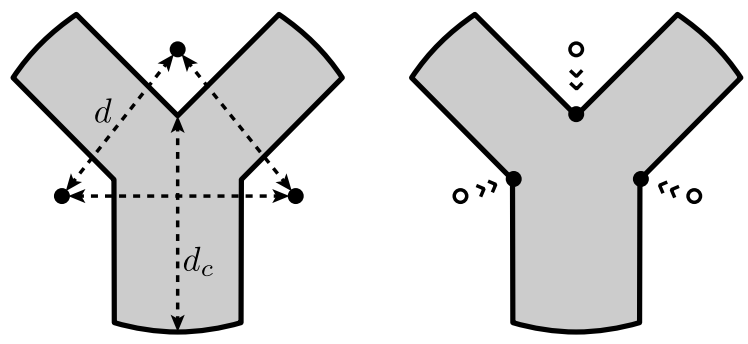

(a)
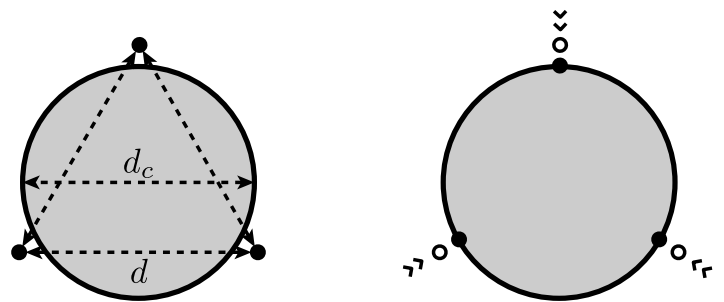

(b)
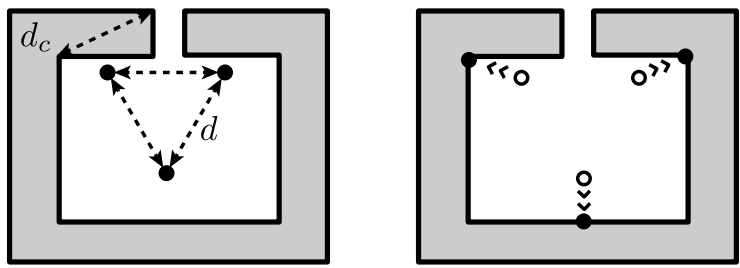

(c)

Fig. 6. Three examples of $F$-cages and possible associated $F$-caging grasps: (a) $F_{e}$-squeezing cage, if initially $F_{e}<2 d_{c}^{2}$, (b) $F_{\max }$-squeezing cage if initially $F_{\max }<d_{c}$, (c) $F_{\min }$-stretching cage if initially $F_{\min }>d_{c}$.

strategies used in [1] by Pereira, Campos and Kumar to cage an object. In that work, they guarantee caging by keeping the interdistances between independent mobile robots below a certain safety threshold. This is, by definition, an $F_{\max }$ squeezing cage, except that they only kept track of distances between adjacent robots in a circular formation, which can also be proven to be a suitable $F$ function.

Guaranteeing caging by keeping track of the values of $F_{e}$ and $F_{\max }$ is also very similar to the concepts of dispersion and concentration proposed by Pipattanasomporn, Vogmasa and Sudsang [13] for caging rigid polytopes. They also consider only distances between adjacent robots in a circular formation.

\section{DISCUSSION}

The main idea defended in this paper is that some cages are specially suited as waypoints to grasp an object. Beginning from a pregrasping cage, there is, by definition, a path that leads to a grasping cage such that the object remains caged all the way.

Mimicking the strategy used in two-fingered manipulators to characterize cages with special squeezing and stretching properties, we have formalized its natural generalization to the $n$-fingered case. Given a scalar function $F$ defined on the 
finger formation and satisfying certain properties, there is a set of configurations of the manipulator for which we can guarantee:

1) The object will never escape the reach of the manipulator if we maintain the value of $F$.

2) We can always grasp it just by increasing or decreasing the value of $F$. This process is blind, in the sense that knowledge of the location of the object is not required during the grasping process.

This gives rise to the concepts of $F$-cage, $F$-squeezing cage, and $F$-stretching cage, configurations of the manipulator that trap the object when given the freedom of moving while maintaining, decreasing or increasing the value of $F$ correspondingly. These generalize the conventional definitions of cage, squeezing cage and stretching cage for the case of two fingers.

In this paper we show that all $F$-cages of an object homeomorphic to an Euclidean $n$-ball are either $F$-squeezing, $F$-stretching, or both. An important consequence is that, when $F$ satisfies the hypothesis in Corollary 1 , all $F$-cages are pregrasping cages, and therefore are useful waypoints to grasp an object.

$$
\text { Cages } \supsetneq \text { Pregrasping cages } \supsetneq F \text {-cages }
$$

As illustrated in (9), the set of cages that ease the grasping problem (pregrasping cages) is a proper subset of the set of all cages. For a specific choice of $F$, we have characterized a set of cages that is fully contained in the set of pregrasping cages, and indirectly given sufficient conditions for a cage to be a pregrasping cage.

\section{APPENDIX A \\ $F$-SqueEZING AND $F$-STRETCHING TheOREM}

Theorem ( $F$-Squeezing/F-Stretching Caging). Let $F$ be $a$ semialgebraic function defined on the point finger formation. Then, given an F-caging configuration of a topological ball in $\mathbb{R}^{d}$, it is either $F$-squeezing caging, F-stretching caging, or both.

Proof: We will prove the contrapositive. Suppose that a certain configuration $c$ of the manipulator is neither $F$ squeezing caging nor $F$-stretching caging. This implies the existence of two escaping paths $\alpha^{+}$and $\alpha^{-}$both living in $\mathcal{M}_{k}^{+}$and $\mathcal{M}_{k}^{-}$to configurations $c^{+}$and $c^{-}$respectively, where $k=F(c)$. The structure of the proof is:

1) Use $\alpha^{+}$and $\alpha^{-}$to build a closed contractible curve in $\mathcal{M}^{\text {free }}$. The contractible curve will necessarily cross $\mathcal{M}_{k}$. We want those crossings not to be $F$-caging.

2) We will construct a path from $c$ to one of such crossings by intersecting the contraction of the curve with $\mathcal{M}_{k}$. This finishes the proof, because that path establishes the non- $F$-cageness of $c$.

Both steps of the process are illustrated in Fig. 5.

In order to build the contractible curve we need to make use of the following proposition:
Proposition 1 (Characterization of contractible paths). $A$ closed path $\alpha$ in $\mathcal{M}^{\text {free }}$ is contractible if and only if for each $i, \Pi_{i}(\alpha)$ describes a contractible path in $\mathcal{W}_{p_{i}}^{\text {free }}$.

The proof can be found in [10]. By Proposition 1, if we want to construct a contractible curve, we can close the path for each point finger independently. Given that $\alpha^{+}$and $\alpha^{-}$are escaping paths, we can suppose that they end up far enough from the object $\mathcal{O}$ so that the section that closes the path is entirely composed of configurations that do not $F$-cage the object. As illustrated in Fig. 7, that is always possible if the final configurations $c^{+}$and $c^{-}$of escaping paths have both point fingers outside a ball $B \supset \mathcal{O}$ with diameter $d_{B}>\frac{n \cdot d}{\pi}$ where $d$ is the diameter of the object.

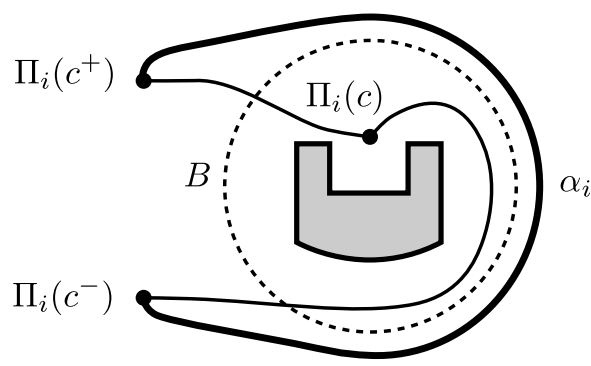

Fig. 7. Completion of the contractible curve in the workspace of point finger $p_{i}$. In the case of dimension 2 we have to chose $\alpha_{i}$ outside the ball $B$ such that the resultant winding number of the closed curve $\Pi\left(\alpha^{+}\right) \oplus \alpha_{i} \oplus \Pi\left(\alpha^{-}\right)$ is zero.

Each point finger $p_{i}^{+}$defined by $c^{+}$must be joined with the corresponding point finger $p_{i}^{-}$defined by $c^{-}$outside $B$ and in a contractible way. This is always possible due to Schoenfliess theorem which characterizes the exterior of a topological ball. When the dimension of the workspace is 2 , we have to follow the boundary of $B$ and turn enough times to undo the winding number of $\Pi_{i}\left(\alpha^{+} \oplus \alpha^{-}\right)$. Lets call $\alpha$ to such a completion curve and $\beta$ to the resultant contractible curve $\alpha^{+} \oplus \alpha \oplus \alpha^{-}$.

The contractibility of $\beta$ implies the existence of an homotopy $H:[0,1] \times[0,1] \longrightarrow \mathcal{M}^{\text {free }}$ that contracts the closed path to $c$. The boundary of the square $S=[0,1] \times[0,1]$ maps into the curve $\beta$, with the inconvenience that three of the sides of the square map into $c$. To avoid this, we consider the quotient map $\pi$ that identifies them into a single point in the boundary of $S$. The map $\pi$ transforms $S$ into a disc $D$ and the quotient topology induces a continuous map $\tilde{H}: D \longrightarrow \mathcal{M}^{\text {free }}$. From now on when mentioning the contraction we will refer to the quotient version $\tilde{H}$ of the homotopy. We will call $q$ to the point in the boundary of $D$ that maps into $c$.

The path that allows $c$ to escape inside $\mathcal{M}_{k}$ lives in $\tilde{H}(D) \cap$ $\mathcal{M}_{k}$. The construction of that intersection relies on the next Lemma, borrowed from [15]:

Lemma 1. Let $M$ be an m-dimensional manifold and $N$ an $n$ dimensional manifold, with $m \geq n$. If $f: M \longrightarrow N$ is smooth, and if $y \in N$ is a regular value, then the set $f^{-1}(y) \subset M$ is a smooth manifold of dimension $m-n$. 
If $M$ is a manifold with boundary $\partial M$ and $y$ is also regular for the restriction $f \mid \partial M$, then $f^{-1}(y)$ is a smooth $(m-n)$ manifold with boundary. Furthermore, the boundary $\partial\left(f^{-1}(y)\right)$ is precisely equal to the intersection of $f^{-1}(y)$ with $\partial M$.

Let $f$ be the composition $f: D \stackrel{\tilde{H}}{\longrightarrow} M^{\text {free }} \stackrel{F}{\longrightarrow} \mathbb{R}$. Then $f^{-1}(k)$, as a subset of $D$, is mapped by $\tilde{H}$ into the mentioned intersection $\tilde{H}(D) \cap \mathcal{M}_{k}$. Proving the existence of the escaping path in $\mathcal{M}_{k}$ is equivalent then to show the existence of a path in $f^{-1}(k)$ from $q$ to another point $q_{0}$ in the boundary of $D$. By construction, $\tilde{H}$ will map that path into an escaping path in $\mathcal{M}_{k}$ to a non- $F$-caging configuration.

If $f$ is smooth and $k$ is a regular value, the application of Lemma 1 says that $f^{-1}(k)$ is a one dimensional smooth manifold with $f^{-1}(k) \cap \partial D$ as boundary. As a consequence, $q$ is connected through a continuous and smooth path to a different point in $\partial D$ within the level set $f^{-1}(k)$, which finishes the proof. However, $f$ is not necessarily smooth neither $k$ a regular value.

In order to grant the smoothness of $f$ we $\varepsilon$-approximate it. This approximation needs to guarantee that the contraction remains in $\mathcal{M}^{\text {free }}$ and that all crossings of the contractible path $\beta$ trough $\mathcal{M}_{k}$ still remain non- $F$-caging. The details are long and we will skip them here for lack of space, but, using an approximation theorem, we can find an algebraic approximation that satisfies both constraints. A similar process is detailed in [10] for the two-finger case.

The other problem to solve is what happens when $k$ is not a regular value of $f$. If that is the case, we can use Sard's Theorem which characterizes the critical points of smooth functions.

Lemma 2 (Sard's Theorem). Let $f: U \longrightarrow \mathbb{R}^{p}$ be a smooth map, with $U$ open in $R^{n}$ and let $C$ be the set of critical points; that is the set of all $x \in U$ with rank $d f_{x}<p$. Then $f(C) \subset \mathbb{R}^{p}$ has measure zero.

The lemma says that the set of regular values is dense on the image of $f$. Consequently, if $k$ is critical, there is a monotonic sequence of regular values $\left\{k_{n}\right\}_{n}$, converging to $k$.

By virtue of Lemma $1,\left\{f^{-1}\left(k_{n}\right)\right\}_{n}$ is a series of smooth one dimensional manifolds. This level sets induce a sequence of smooth paths that gradually approach the level set $f^{-1}(k)$, as illustrated in Fig. 8. In general, the limit of a sequence of continuous paths does not necessarily converge to a continuous path but, using the previous semialgebraic approximation of the contraction, the function $f=F \circ \tilde{H}$, level sets $\left\{f^{-1}\left(k_{n}\right)\right\}_{n}$ and $f^{-1}(k)$ will also be semialgebraic. Consequently, the approximating paths and the limit, that lives in a semialgebraic set, must also be continuous and semialgebraic. Which concludes the proof.

\section{ACKNOWLEDGMENT}

This work was sponsored in part by the Army Research Laboratory under Cooperative Agreement Number W911NF10-2-0016, DARPA, and NSF. The views and conclusions

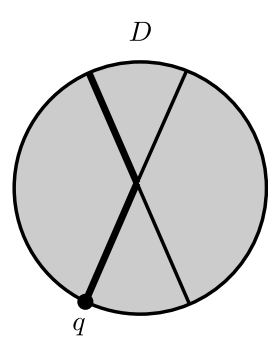

(a)

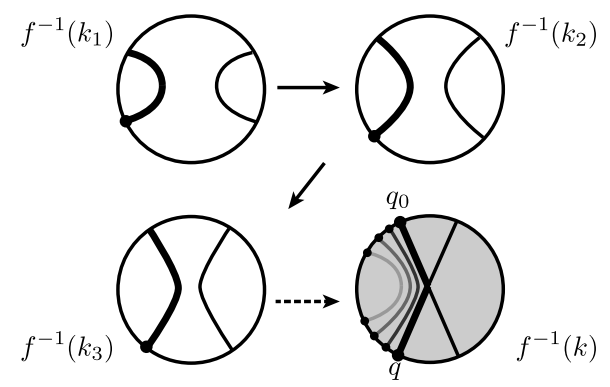

(b)
Fig. 8. (a) When $k$ is a critical value, $f^{-1}(k) \subset D$ might not be a smooth manifold. Still $q$ is path-connected to a point $q_{0} \in \partial D$ mapped by $\tilde{H}$ to a noncaging configuration. (b) To prove it we consider the limit of a series of level sets $\left\{f^{-1}\left(k_{n}\right)\right\}_{n}$ of regular points where the path is guaranteed.

contained in this document are those of the authors and should not be interpreted as representing the official policies, either expressed or implied, of the Army Research Laboratory, DARPA, or the U.S. Government.

\section{REFERENCES}

[1] G. Pereira, M. Campos, and V. Kumar, "Decentralized Algorithms for Multi-Robot Manipulation via Caging," International Journal of Robotics Research, vol. 23, no. 7-8, pp. 783-795, 2004.

[2] S. J. Blind, C. C. McCullough, S. Akella, and J. Ponce, "Manipulating Parts with an Array of Pins: A Method and a Machine," International Journal of Robotics Research, vol. 20, no. 10, pp. 808-818, 2001.

[3] A. Sudsang and J. Ponce, "A New Approach to Motion Planning for Disc-Shaped Robots Manipulating a Polygonal Object in the Plane," in IEEE International Conference on Robotics and Automation, vol. 2, no. April, 2000, pp. 1068-1075.

[4] M. Vahedi and A. F. van Der Stappen, "Caging Polygons with Two and Three Fingers," The International Journal of Robotics Research, vol. 27, no. 11-12, pp. 1308-1324, Nov. 2008

[5] W. Kuperberg, "Problems on polytopes and convex sets," in DIMACS: Workshop on polytopes, 1990 , pp. 584-589.

[6] A. Sudsang, J. Ponce, and N. Srinivasa, "Grasping and In-Hand Manipulation: Geometry and Algorithms," Algorithmica, vol. 26, pp. 466-493, 1999.

[7] R. Diankov, S. S. Srinivasa, D. Ferguson, and J. Kuffner, "Manipulation Planning with Caging Grasps," in IEEE International Conference on Humanoid Robots, 2008.

[8] P. Pipattanasomporn and A. Sudsang, "Two-finger Caging of Concave Polygons," in IEEE International Conference on Robotics and Automation. IEEE, 2006, pp. 2137-2142.

[9] E. Rimon and A. Blake, "Caging Planar Bodies by One-Parameter Two-Fingered Gripping Systems," The International Journal of Robotics Research, vol. 18, no. 3, pp. 299-318, Mar. 1999.

[10] A. Rodriguez and M. T. Mason, "Two Finger Caging: Squeezing and Stretching," in Algorithmic Foundations of Robotics (WAFR). Springer, 2008.

[11] C. Davidson and A. Blake, "Error-tolerant Visual Planning of Planar Grasps," in International Conference on Computer Vision, 1998, pp. 911-916.

[12] K. G. Gopalakrishnan and K. Goldberg, "Gripping Parts at Concave Vertices," in IEEE International Conference on Robotics and Automation, 2002, pp. 1590-1596.

[13] P. Pipattanasomporn, P. Vongmasa, and A. Sudsang, "Caging rigid polytopes via finger dispersion control," 2008 IEEE International Conference on Robotics and Automation, pp. 1181-1186, May 2008.

[14] E. Rimon and J. W. Burdick, "On force and form closure for multiple finger grasps," Proceedings of IEEE International Conference on Robotics and Automation, no. April, pp. 1795-1800, 1996.

[15] J. W. Milnor, Topolgy from the Differentiable Viewpoint. Princeton University Press, 1965. 